Casos Clínicos

Arch. Esp. Urol., 60, 1 (69-71), 2007

\section{SÍNDROME DE GOLDENHAR ASOCIADO A VÁLVULAS DE LA URETRA POSTERIOR.}

Alberto Palacios, António Pedro Carvalho, Ribeiro de Castro y Armando Reis.

Servicio de Urología Pediátrica. Hospital María Pía. Porto. Portugal.

Resumen.- OBJETIVO: Presentamos un caso con síndrome de Goldenhar con válvulas de la uretra posterior.

MÉTODO: El Síndrome de Goldenhar (displasia oculoaurículovertebrall es una entidad infrecuente que se caracteriza por anomalías: oculares (dermoides epibulbares, colobomas), auriculares (baja implantación auricular, apéndices preauriculares) y/o anomalías vertebrales, asociado a múltiples malformaciones cardíacas, cerebrales y renales.

RESULTADOS: Realizamos electrofulguración de las válvulas de la uretra posterior, teniendo buena evolución.

CONCLUSIONES: Los niños nacidos con Síndrome de Goldenhar deberían someterse a pruebas de imagen para detectar anormalidades del aparato urinario superior e inferior.

Palabras clave: Síndrome de Goldenhar. Displasia oculoaurículovertebral.
Summary.- OBJECTIVE: To report a case with Goldenhar syndrome with posterior urethral valves.

METHODS: Goldenhar syndrome loculoauriculovertebral dysplasial is a condition featuring the following triad of anomalies: ocular abnormalities lepibulbar dermoids, coloboma), otic anomalies (low set ears, auricular appendage) and/or vertebral anomalies. Multiple malformations, including congenital heart, brain and renal disease.

RESUITS: Posterior urethral valves should be treated with primary valve ablation.

CONCLUSIONS: It is necessary to perform a careful evaluations of general malformations, especially renal malformations.

Keywords: Goldenhar syndrome. Oculoauriculovertebral dysplasia.

\section{INTRODUCCIÓN}

El síndrome de Goldenhar es una variante de displasia oculoauriculovertebral, que presenta una amplia variedad de anomalías caracterizadas por una combinación de microsomia hemifacial y otras alteraciones como dermoides epibulbares o lipodermoides, micrognatia, apéndices auriculares, hipoplasia mandibular, alteracionesvertebrales y malformaciones en múltiples órganos(1), incluyendo el aparato urinario. Embriológicamente, el defecto oculoaurículovertebral trata de una alteración del primero y segundo arco braquial (2), aunque esta anomalía no explica las anomalías que aparecen en el cerebro, corazón, riñón o médula (3).

\section{CASO CLÍNICO}

Lactante, de raza caucasiana, la primera hija de padres jóvenes (madre de 28 años y padre de 30 años) sanos y no consanguíneos, sin historia de patología heredofamiliar, renal ni hipertensión conocida. Gestación correctamente vigilada y sin alteraciones. Parto eutócico a las 37 semanas sin complicaciones, presentando un test de Apgar de 9/10. Antropometría al nacimiento, peso de 2.760 gramos, con diámetro céfalo-caudal de 48,5 $\mathrm{cm}$ y perímetro cefálico de $34,7 \mathrm{~cm}$. VDRL, AntHBs, VIH negativos, Toxoplasmosis no inmune y rubéola inmune. Detectados apéndices auriculares bilaterales, baja implantación del pabellón auditivo (Figura 1), granulomas cutáneos, micrognatismo con hipoplasia mandibular (Figura 2), coloboma localizado en el ojo izquierdo (Figura 3) y tumefacción escrotal sólida aparentemente no dolorosa a la palpación. Calendario de vacunas actualizado.

El lactante es transferido de otro hospital a los 19 días de vida con el diagnóstico de urosepsis. Internado inicialmente en la Unidad de Cuidados Intensivos Neona- 


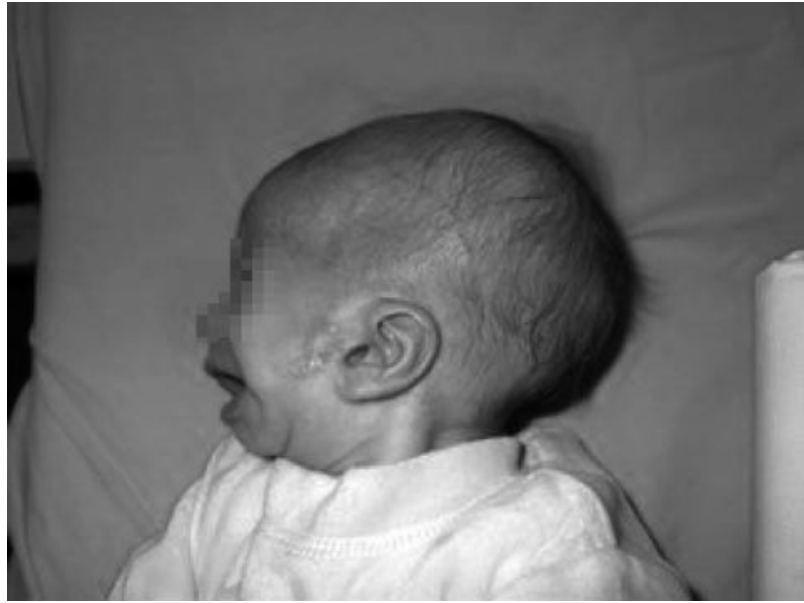

FIGURA 1. Implantación baja del pabellón auditivo. Presencia de cicatrices posterior a la exéresis de los apéndices auriculares.

tales para estabilizar el cuadro, mediante medidas de soporte y antibioterapia con ampicilina, gentamicina y cefotaxima.

Realiza ecografia reno-pélvica- escrotal que revela uretero-hidronefrosis bilateral y vejiga de paredes espesadas, tumefacción escrotal derecha, con aparente imágenes hipo e hiperecogénicas con septos y aparente destrucción testicular.

Una vez que se estabilizan los parámetros y con urocultura negativa, realizamos una cistouretrografía miccional seriada presentando una dilatación de la uretra posterior, vejiga trabeculada y sin presencia de reflujo vésico-ureteral, sospechando cuadro de válvulas de la uretra posterior (Figura 4).

Las radiologías óseas de cuerpo entero no detectan alteraciones óseas, ni alteraciones vertebrales.

Realizamos fulguración de las válvulas de la uretra posterior y orquidectomía inguinal derecha. El postoperatorio

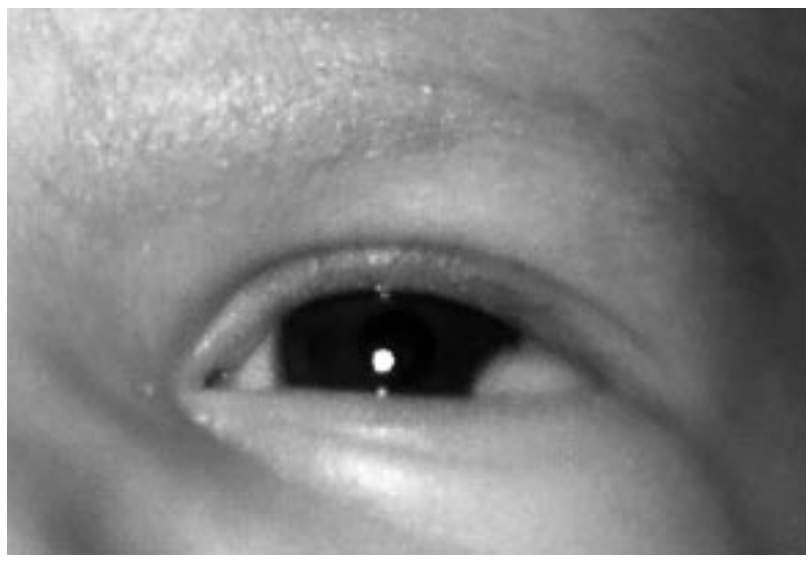

FIGURA 3. Coloboma.

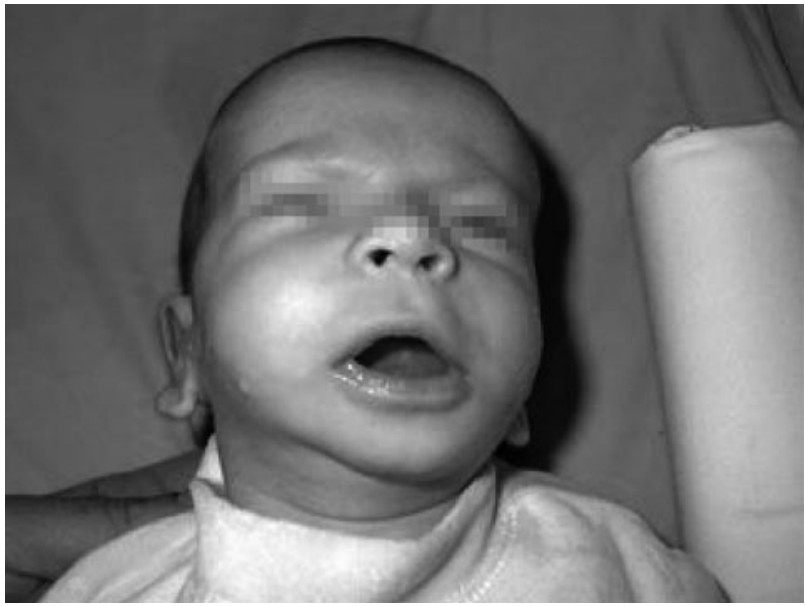

FIGURA 2. Hipoplasia mandibular.

transcurre sin complicaciones. Retiramos la sonda al séptimo día del postoperatorio. Es dado de alta con función renal normal, urocultura negativa y correcta diuresis.

Al cuarto mes de vida es internado con el diagnóstico de infección respiratoria en el contexto de bronquiolitis,

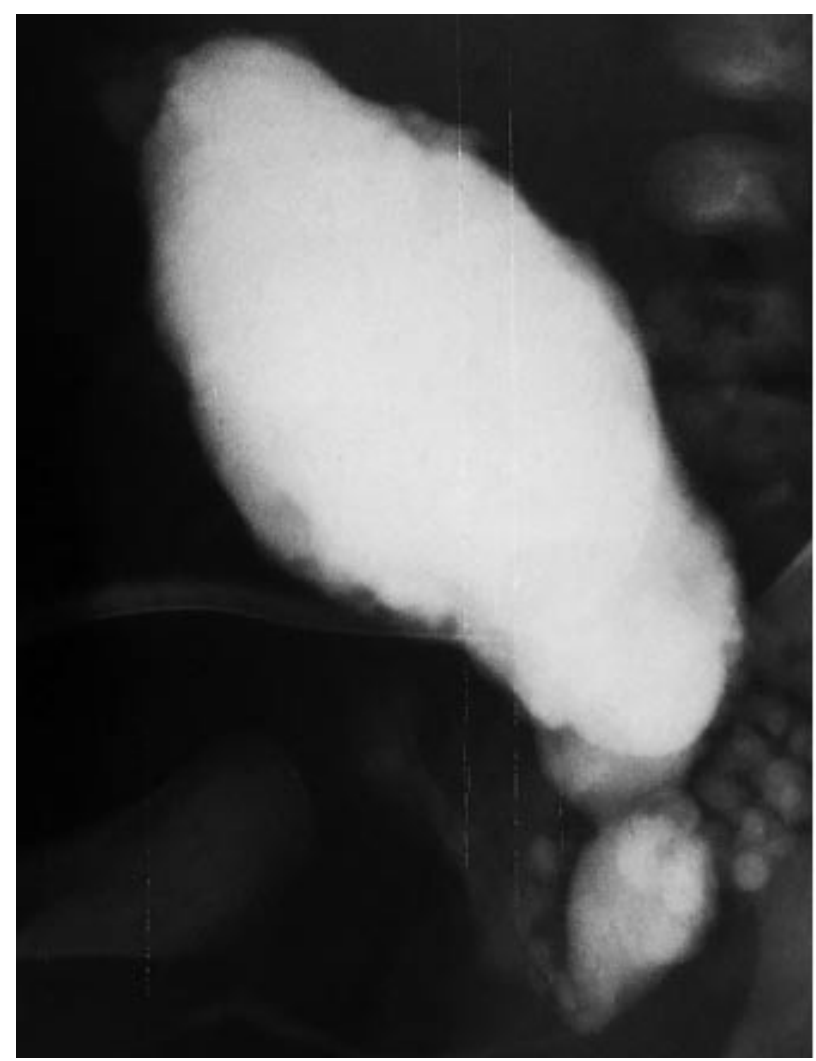

FIGURA 4. Vejiga trabeculada, dilatación de la uretra posterior, relujo a los canales eyaculadotes y válvulas de la Uretra posterior. 
con uroculturas positivas para flora polimicrobiana es medicado con antibioterapia, y dado de alta con profilaxia antibiótica con trimetroprim-sufametoxazol.

Al quinto mes teniendo una orina aséptica, realizamos urografía endovenosa y cintilograma renal con MAG3 y prueba de furosemida, presentando dilatación ureteral bilateral, bifidez renal derecha, con capacidad vesical normal y con vaciado completo de la vejiga, uretra aparentemente sin alteraciones, la función renal bilateral está conservada, respondiendo a la prueba de furosemida. Llegamos al diagnóstico de dilataciones ureterales bilaterales no obstructivos asociados a bifidez piélica. Decidimos efectuar controles cada 6 meses.

En el último control el niño con 15 meses de edad, presenta función renal normal, buena diuresis y sedimentos urinarios sin alteraciones.

\section{DISCUSIÓN}

El síndrome de Goldenhar es parte de una entidad patológica oculo-aurículo-vertebral y es considerado un síndrome por que, a parte de presentar displasia mandibulofacial, existen con frecuencia alteraciones vertebrales.

Estudios en animales de experimentación sugieren que el síndrome de Goldenhar aparece por causa de una disrupción vascular durante la embriogénesis, a los 35 a 40 días de gestación. Esta alteración vascular impide la morfogénesis correcta de las estructuras derivadas del primer y segundo arco braquial, resultando en las alteraciones que aparecen en el nacimiento $(4,5)$.

Los niños pueden presentar hipoplasia de la musculatura facial, micrognatia, apéndices preauriculares y displasia del pabellón auditivo, hipoplasia de las vértebras a nivel cervical, torácico o lombar, dermoides epibulbares, microftalmia, colobomas, labio leporino y alteraciones orgánicas cardíacas, renales y del sistema nervioso central. Existen por lo tanto gran variedad de presentaciones clínicas, de hecho la mayoría de los niños se presentan con asimetría facial y displasia del pabellón auditivo externo (4).

La incidencia de esta patología es aproximadamente uno de cada 5600 recién nacidos (4). La mayoría de los casos aparecen de una manera esporádica, aunque ya se han publicado casos con herencia autonómica dominante, y otros en el que existía consanguinidad entre los padres, lo que hace suponer un herencia autonómica recesiva (5). Se han reportado casos de gemelos monocigóticos donde uno de los niños presenta el síndrome de Goldenhar y el otro es sano.

El síndrome es causado por alteraciones del primer y segundo arco braquial. Aunque esa teoría no puede explicar las anormalidades vertebrales, ano-vaginales, del aparato urinario que tiene un origen embriológico diferente al primer y segundo arco braquial. Russell y cols. proponen que el síndrome puede ser el resultado de una deficiencia del mesodermo causado por una alteración en la migración celular, ya que estos órganos afectados en el síndrome son derivados del mesodermo (6).

Existen pocos artículos en la literatura que hagan referencias a anomalías del aparato urinario. Según algunos autores el porcentaje de alteraciones en el aparato urinario rondan el 5\%. La mayoría de las alteraciones son encontradas de una manera incidental, y la malformación mas frecuente encontrada es la ectopia y fusión renal, seguida de la agenesia renal y del reflujo vésico-ureteral. Según Ritchey y cols. de los 28 niños con síndrome de Goldenhar 20 presentaron anomalías del tracto urinario lo que supone un $70 \%$ de los casos. Este autor afirma que todos los casos con este síndrome deberían someterse e exámenes de imagen para descartar anomalías del tracto urinario (7). Nuestro paciente presentaba válvulas de la uretra posterior, anomalía infrecuentemente asociada a este síndrome.

\section{CONCLUSIÓN}

El Síndrome de Goldenhar es infrecuente. No existe determinada la incidencia de alteraciones en el aparato urinario. Nosotros diagnosticamos un niño con síndrome de Goldenhar asociado a bifidez renal y válvulas de la uretra posterior. Coincidimos con otros autores en que estos niños se deben someter a exámenes de imagen para descartar posibles malformaciones del aparato urinario superior e inferior.

\section{BIBLIOGRAFIA y LECTURAS RECOMENDADAS (*lectura de interés $y^{* *}$ lectura fundamental)}

*1. GORLIN, R.J.; JUE, K.L.; JACOBSEN, U. Y cols.: "Oculoauriculovertebral dysplasia". J. Pediatr.;63:991. 1963.

2. VAN BEVER, Y.; VAN DEN ENDE, J.J.; RICHIERI-COSTA, A.: "Oculo-auriculo-vertebral complex and uncommon associated anomalies: Report on 8 unrelated Brazilian patients". Am. J. Med. Genet;44:683. 1992.

3. SUGAR, H.S.: "The oculoauriculovertebral dysplasia syndrome of Goldenhar". Am. J. Ophtalmol.;62:678. 1966.

**4. GORLIN, R.L.: "Branchial arch and oro-acral disrosders". In: Gorlin, J.J.; Cohen, Jr. M.M.; Hennekam, R.C., editors. "Syndromes of the head and neck". London: Oxford University Press, p. 790. 2001.

5. REGENBOGEN, L.; GODEL, V.; GOYA, V. y cols.: "Further evidence for an autosomal dominant form of oculoaruiculovertebral dysplasia". J. Pediatr. 1986, Clin Genet.;21:161. 1982

6. RUSSELL, L.J.; WEAVER, D.D.; BULL, M.J.: "The axial mesodermal dysplasia spectrum". Pediatrics;67:176. 1981.

*7. RITCHEY, M.L.; NORBECK, J.; HNANG, C. y cols.: "Urologic manifestations of Goldenhar syndrome”. Urology Jan;43:88. 1994 